\title{
Long-term outcome with lenalidomide and dexamethasone therapy for newly diagnosed multiple myeloma
}

\author{
G Srivastava, V Rana, MQ Lacy, FK Buadi, SR Hayman, A Dispenzieri, MA Gertz, D Dingli, S \\ Zeldenrust, S Russell, A McCurdy, P Kapoor, R Kyle, SV Rajkumar, and S Kumar \\ Division of Hematology and Blood and Marrow Transplant, Department of Medicine, Mayo Clinic, \\ Rochester, MN, USA
}

\begin{abstract}
The combination of lenalidomide and dexamethasone (Len-Dex) is a commonly used initial therapy for newly diagnosed multiple myeloma (MM). Although the initial response rates and toxicity are well known, long-term outcome is not well described. We studied 286 consecutive patients with newly diagnosed MM initially treated with Len-Dex. The median (range) age at diagnosis was $63(28-92)$ years, $166(58 \%)$ patients 55 years and $175(61 \%)$ male. The median estimated duration on Len-Dex was 5.3 months with overall response ( partial response) of $72 \%$, including $26 \%$ with very good partial response or better. The median overall survival (OS) from the diagnosis was not reached (NR) and the estimated 5-year survival was $71 \%$. The median time to first disease progression, irrespective of transplant status, was 30.2 months. Overall, 143 (50\%) patients underwent stem cell transplant. The median OS was NR for patients $\leq 70$ years and 5.8 years for the older patients $(P=0.01)$. The 5 -year OS estimate for patients in International Staging System stage 1, 2 and 3 were 82,65 , and $44 \%$ respectively. There were 21 new second malignancies after MM diagnosis $(6.6 \%)$. The median survival exceeding 7 years reflects the efficacy of novel agents. The risk of second malignancies doesn't appear to be excessive in this population
\end{abstract}

\section{Keywords}

multiple myeloma; lenalidomide; long-term outcome; secondary malignancy

\section{INTRODUCTION}

Over the past decade, survival of patients with multiple myeloma (MM) has improved dramatically with the advent of novel classes of effective drugs, such as the proteasome inhibitors and the immunomodulatory drugs. ${ }^{1}$ On the basis of the promising results seen with these drugs in the treatment of newly diagnosed myeloma in phase $2^{2,3}$ and phase 3

\footnotetext{
(C) 2013 Macmillan Publishers Limited All rights reserved

Correspondence: Dr S Kumar, Division of Hematology and Blood and Marrow Transplant, Department of Medicine, Mayo Clinic, Rochester, MN 55905, USA. kumar.shaji@mayo.edu.

CONFLICT OF INTEREST

$\mathrm{AD}$ received research dollars from Celgene, Millenium, Pfizer, MAG received honoraria for lectures from Celgene, RK is on the monitoring committees for Celgene as well as their advisory board for secondary malignancies, SVK received research support for clinical trials form Celgene, Millennium, Novartis, Cephalon, Onyx, consulting Merck, Celgene, Onyx.

AUTHOR CONTRIBUTIONS

GS, VR, SK designed the research, collected and analyzed the data, and wrote the manuscript; MQL, FKB, SRH, AD, MAG, DD, SZ, SR, AM, PK, RK, SVR assisted in data collection and manuscript preparation; and all the authors approved the final draft of the manuscript.
} 
trials, ${ }^{2,4-6}$ combinations that include one or more of these drugs have become the standard initial therapy for MM. ${ }^{7}$ Although the initial trials examined the efficacy of thalidomide, lenalidomide and bortezomib in combination with corticosteroids, more recently several large phase 3 trials $^{8-11}$ have evaluated multidrug combination of the novel drugs used together or in combination with alkylating agents or anthracyclines. Despite significantly improved responses seen with these combinations, ${ }^{12}$ studies up until now have not shown any improvement in overall survival (OS) with the multidrug combinations compared with the novel drug-dexamethasone combinations. One of the disadvantages of these studies has been the lack of long-term follow up given the recent nature of these trials. Furthermore, interpretation of long-term results is clouded by the heterogeneity in treatment in terms of use of stem cell transplant along with variable use of consolidation and maintenance strategies.

Among these novel agents combinations, lenalidomide and dexamethasone (Len-Dex) is a commonly used initial therapy for newly diagnosed MM. There have been two large phase three trials done with this combination in the setting of newly diagnosed myeloma; one comparing the combination with dexamethasone alone ${ }^{13}$ and the second trial ${ }^{4}$ comparing two different doses of dexamethasone in combination with lenalidomide. Although the former trial showed improved responses with the Len-Dex combination before stem cell transplant, no long-term results are available in terms of OS and potential issues with cumulative toxicities. The latter trial showed that lenalidomide in combination with lowdose (weekly) dexamethasone was associated with reduced early mortality, but again no long-term follow up is available as it was designed as an induction trial to assess response before transplant. Furthermore, the risk of second malignancies has been raised as a concern with lenalidomide therapy, as was described in three large recently reported randomized trials using lenalidomide maintenance after initial induction ${ }^{14}$ or after autologous transplant. ${ }^{15,16}$ Given these factors, data regarding long-term outcome of initial therapy with lenalidomide and dexamethasone will be helpful in deciding on treatment choices for these patients.

\section{PATIENTS AND METHODS}

\section{Patients}

We retrospectively analyzed the medical records of 305 consecutive patients seen at Mayo Clinic, Rochester with newly diagnosed MM, who received initial treatment with the LenDex regimen. The study included patients seen between January 2003 and January 2011, of whom 286 consecutive patients had complete follow up records, and were included in the current analysis. Information pertaining to baseline patient characteristics, laboratory data at diagnosis and data regarding the use of lenalidomide and second malignancies was obtained from the patient database and examination of medical records. All patients had given informed consent for the use of their records for research purposes, and the study was conducted in accordance with the institutional guidelines with approval of the Institutional Review Board of the Mayo Clinic and in accordance with the Declaration of Helsinki.

\section{Treatment}

Patients were treated with lenalidomide $25 \mathrm{mg}$ daily for 21 days of a 28 -day cycle. Initial patients were treated with high-dose dexamethasone (40mg on days 1-4, 9-12, 17-20 of a 28 -day cycle), which was changed to $40 \mathrm{mg} 6 \mathrm{ial}^{4}$ was reported. Supportive care strategies were according to standard clinical practice and have not changed significantly during the study period. Patients received bone disease therapy with a bisphosphonate, typically pamidronate, consistent with our standard practice. All patients received aspirin for thromboprophylaxis, and those at high risk of thrombotic events received warfarin or low- 
molecular-weight heparin. Antibiotic prophylaxis was not routinely used, and the use of erythropoietic agents was avoided due to the increased risk of thrombosis associated with their use. All patients had disease assessment after each cycle of treatment, and the International Myeloma Working Group (IMWG) uniform response criteria was used to assess disease response. Outside of a clinical trial setting, a bone marrow was not always performed for confirmation of a complete response.

\section{Statistical analyses}

Nominal variables were compared using Fisher's exact test and continuous variables were compared using Student's $t$-test or Wilcoxon signed rank test. Kaplan-Meier method ${ }^{17}$ was used for estimating OS, and progressionfree survival (PFS) and survival curves were compared using log rank test.

\section{RESULTS}

The median age at diagnosis for the study cohort was 63 years (range; 28-92); 166 (58\%) were 565 years, $15(5 \%)$ were over 75 years and $175(61 \%)$ were male. Other baseline characters are described in Table 1 . The median estimated follow-up from diagnosis for the entire cohort was 4.2 years (95\% confidence interval (CI): 4.0, 4.7), and 196 (68\%) patients were alive at the time of last follow up. Among the 286 patients, fluorescence in situ hybridization data were available for $244(85 \%)$ patients. 90\% ( $n=219)$ of patients had fluorescence in situ hybridization abnormalities either at diagnosis or later in the disease course, with $124(51 \%)$ having trisomies and $72(30 \%)$ with translocations involving immunoglobulin heavy chain gene (IgH). Out of 244 patients, $67 \%(n=165)$ had the abnormalities noted at diagnosis, and for the rest of $33 \%(n=79)$ these were noted later during the disease course. High-risk cytogenetics were defined as presence of $t(4 ; 14)$, $\mathrm{t}(14 ; 16)$ and $\mathrm{t}(14 ; 20)$ at any time or $17 \mathrm{p}$ - seen on a fluorescence in situ hybridization at diagnosis. Forty $(16 \%)$ out of 244 patients had high-risk cytogenetics with $t(4 ; 14)$ in 15 , $\mathrm{t}(14 ; 16)$ in 8 , del $17 \mathrm{p}$ in 16 (at diagnosis) and $\mathrm{t}(14 ; 20)$ in 1 patient.

\section{Treatment and response}

Lenalidomide was used in combination with low-dose dexamethasone in $231(81 \%)$ patients, while the remaining patients received high-dose dexamethasone. The median estimated duration of Len-Dex therapy was 5.3 months (95\% CI: 4.6, 6.4), including 93 patients (32\%) who stayed on therapy for 12 months or more. The median duration of therapy was similar for the highdose Dex and the low-dose Dex groups. At the last follow up, $41(14 \%)$ patients were still continuing on therapy, having been on therapy for a median of 30 months (range; 4-93). Two hundred and forty-three patients have gone on to receive additional therapy, including 143 (50\%) patients who have received a stem cell transplant. Among those going to transplant, 130 patients received early transplant in a planned fashion ( $\leq 2$ months from diagnosis), while the others received transplant at the time of relapse from Len-Dex therapy (salvage or delayed autologous stem cell transplantation). The overall response ( \partial response) was $72 \%$, including $15 \%$ with very good partial response and $11 \%$ with a complete response. Out of 286 patients, $14(5 \%)$ were not evaluable for a response either due to missing follow up $\mathrm{M}$ protein values or $\mathrm{M}$ protein measurements at diagnosis not meeting the threshold for measurable disease. Among the patients on therapy for at least 12 months, the objective response rate was $87 \%$, including $45 \%$ with very good partial response or better (Table 2). The proportion of patients with a partial response or better was higher among the high-dose dexamethasone compared with the low-dose Dex group ( 84 vs $69 \% ; P=0.04$ ). The median time to best response among those achieving at least a partial response was 4 months (range; $1-40$ months). Stem cell transplantation was 
the most common reason for discontinuation of Len-Dex therapy (39\%) followed by disease progression or lack of response $(23 \%)$ and toxicity (13\%).

\section{Survival outcomes}

Overall, 184 (64\%) patients have had at least one disease progression since diagnosis. The median time to first disease progression, irrespective of transplant status, was 30.2 months (95\% CI: 25, 42); Figure 1. Censoring those patients who proceeded to stem cell transplantation before relapse at the time of bone marrow transplantation, the median time to progression was 25.5 months (95\% CI: 22, 29). The median OS for the entire cohort from diagnosis was not reached (95\% CI: 6.5, NR (not reached)) and the estimated 5-year survival was $71 \%$ (Figure 1). There were $16(5.5 \%)$ patients who died within the first year after diagnosis. The median OS was 5.8 years (95\% CI: 3.3, NR) for the 59 patients 70 years or older, compared with median not reached (95\% CI: 7.4, NR) for the younger patients $(P=0.002)$ (Figure 2). The five-year survival estimates were $74 \%$ and $57 \%$, respectively, for the patients younger than 70 and the older patient group. The OS was similar for those receiving high-dose Dex compared with the low-dose Dex group. The 5-year OS estimate for patients in International Staging System stage 1, 2 and 3 were 82, 65, and 44\%, respectively (Figure 3). Patient with high-risk cytogenetics had significantly inferior PFS of 1.4 years as compared with 3.4 years among standard risk patients $(P=0.0002)$ (Figure $4 \mathrm{a})$. The 5-year estimated OS was $77 \%$ for standard risk patients as compared with only $47 \%$ for patients with high-risk cytogenetics ( $P=0.0001)$ (Figure $4 \mathrm{~b})$.

\section{Risk of second cancers}

In our study cohort, there were 21 new second malignancies after therapy with lenalidomide (6.6\%); Table 3. These included nine with non-melanoma skin cancers (basal cell or squamous cell), three melanomas, two acute myeloid leukemia, two breast cancers and one each of prostate, sarcoma, papillary thyroid, nasopharyngeal and bladder cancer. This translated to 9 malignancies (12 excluding skin cancers) during 1120 person years of follow up from the diagnosis of myeloma. The median exposure to lenalidomide was five cycles (range; 1-93). The median estimated time to the onset of second cancer was 44 months (95\% CI: 42, 48). Only age at diagnosis significantly affected the risk of a second cancer; those older than 65 had a 2.7 (95\% CI: 1.1, 6.6) fold higher risk for developing a second malignancy (including skin cancer). The estimated duration of lenalidomide therapy among the 21 patients with a second cancer was 10.5 months vs 5.1 months for the remaining patients with no second cancers ( $P$-not significant). More intriguing was the finding of high rate of previous malignancies in this cohort before MM diagnosis with 52 patients (18\%) having one or two prior malignancies before diagnosis of MM. Out of these 52 cancers, 37 were solid tumors.

\section{DISCUSSION}

In the current era of multiple Food and Drug Administration approved novel agents (both immunomodulatory drugs and proteasome inhibitors), there is an ongoing debate in regards to optimal initial regimen for the treatment of MM. More and more patients are being treated with combination regimen of VRD (bortezomib/lenalidomide and dexamethasone) as this regimen results in high response rate, including high complete response rate. ${ }^{18}$ At present there is lack of data indicating any survival benefit for combining all these agents together. In a recently reported study ${ }^{19}$, the OS at 3 years with Len-Dex regimen was similar to three drug regimens, such as combination of CyBorD (bortezomib/cyclophosphamide/ dexamethasone) orVRD. Furthermore, two large randomized trials comparing two versus three drugs regimens in the elderly ${ }^{20,21}$ did not reveal any statistically significant difference in OS. In the study ${ }^{21}$ by HOVON group, three drug regimen of MPT (melphalan/ 
prednisone/thalidomide) had median OS of 40 months, similar to the 45 months of median survival in the MPT arm of the study ${ }^{20}$ by Palumbo et al. ${ }^{22}$ Palumbo et al. also compared a four drug regimen VMPT (bortezomib/melphalan/prednisone/thalidomide) (followed by VT, bortezomib/thalidomide maintenance) to a three drug regimen of VMP in an elderly population (median age of 71 years) deemed ineligible for transplant, and 3-year OS was similar at $89 \%$ and $87 \%$, respectively. This study ${ }^{22}$ did meet its primary end point of improved PFS, with 3-year PFS being 56\% in VMPT-VT arm as compared with $41 \%$ in the VMP arm though at cost of higher grade 3/4 neutropenia as well as cardiac toxicity (10 vs $5 \%$ ). Even in the patients eligible for autologous stem cell transplantation after induction therapy, a large randomized study ${ }^{9}$ comparing three drug regimen VTD (bortezomib/ thalidomide/ dexamethasone) to two drug regimen of TD (thalidomide/ dexamethasone) did not show any difference in survival (3-year OS of $86 \%$ in VTD vs $84 \%$ in TD arm). Our study demonstrates that with the Len-Dex regimen median survival exceeds 7 years and median OS in patients $>70$ years in our study was 5.8 years. This is similar if not better compared with other previously reported regimens in elderly patients. ${ }^{20,21,23}$ The Len-Dex regimen minimizes the toxicity as patients are not exposed to all active agents upfront at diagnosis. In the era of novel drugs, MM is evolving to a chronic disease, and we do need to preserve active agents to be used in a sequential manner at the time of disease relapse. Improved prolonged PFS with the use of multiple agent at diagnosis has not resulted in OS benefit as of yet. 24

Our study provides long-term estimates of responses and survival in a series of patients treated initially with lenalidomide and dexamethasone. The median survival exceeding 7 years reflects the efficacy of the novel agents both at diagnosis and at relapse, and confirms the survival improvements seen in MM in the last decade. Len-Dex is also fairly well tolerated as $32 \%$ of patients remained on therapy for 12 months or more. At the last follow up, 41 (14\%) patients were still continuing on therapy, having been on therapy for a median of 30 months. Patient with high-risk cytogenetics had worse OS and PFS as compared with the standard risk patients, and this is consistent with recent studies ${ }^{25,26}$ reported in the era of novel therapies.

The rate of occurrence of second solid malignancies in our cohort is comparable to rates recently reported in three large randomized trials involving Len therapy. In the study by Palumbo et al. ${ }^{14}$ the 3 -year risk of second invasive malignancy was $7 \%$ in melphalan, prednisone, lenalidomide followed by lenalidomide maintenance, and was $3 \%$ in the melphalan plus prednisone arm. In our study the incidence rate was $3.5 \%$ for second solid malignancies as compared with $3.3 \%{ }^{16}$ and $4.3 \%,{ }^{15}$ respectively, in two lenalidomide maintenance trials after autologous stem cell transplant. In terms of second hematological malignancies, our study had a lower rate of $0.7 \%$ as compared with $4.2 \%$ and $3.5 \%$, respectively, in the studies by Attal et al. ${ }^{16}$ and McCarthy et al. ${ }^{15}$ The incident rate of second malignancies in our study is also comparable to what was observed in a pooled analysis of 11 clinical trials of lenalidomide-based therapy for relapsed/refractory MM. ${ }^{27}$ The relatively low risk seen in our experience is likely a reflection of timing of the use of lenalidomide. The studies where the increased risk has been reported have been post highdose melphalan or in combination with melphalan. In our cohort, only $50 \%$ of the patients had undergone stem cell transplant. As we are dealing with small numbers of second malignancies, it is very hard to draw any firm conclusions. In this cohort, we identified a high rate of second malignancies even before diagnosis of MM. There were 40 (14\%) malignancies (excluding non-melanoma skin cancers) occurring before the diagnosis and treatment of MM, suggesting that their occurrence is secondary to inherent risk among these patients. This is further corroborated by the observations made even before the approval of lenalidomide, that risk of second malignancies is much higher in patients with MM as compared with general population. ${ }^{28}$ Recently even monoclonal gammopathy of 
undetermined significance was reported to be associated with an eightfold increased risk of acute myeloid leukemia/myelodysplastic syndrome,${ }^{29}$ suggesting the risk of second malignancies is likely primarily related to host and disease (MM) related factors. In addition, with the increased survival of patients with MM in the last decade, the patients are now living long enough to develop second malignancies, which we would not have noticed in the past due to short survival. Lastly, the risk of death due to progressive MM remains much higher than due to second malignancies, as was evident in our cohort with only one patient dying due to second malignancy (acute myeloid leukemia) developing after MM diagnosis.

Limitations of our study include those common to retrospective studies using data abstracted from patient records. These data sources tend to capture incomplete information regarding toxicity. The major strength of this study is the large sample size of patients who were treated homogenously according to consensus guidelines set forth by our group.

In conclusion, this study demonstrates that long-term outcomes with Len-Dex regimen results in median survival exceeding 7 years, is associated with superior results in the elderly, and the risk of second malignancies does not appear to be excessive in the context of primary therapy with lenalidomide dexamethasone combination.

\section{Acknowledgments}

This work was supported in part by Mayo Clinic Hematological Malignancies Program, Paul Calabresi K12 Award (CA96028). Also supported in part by grants CA 107476, CA 62242, CA100707 and CA 83724 from the National Cancer Institute, Rockville, MD, USA, and also supported in part by the Jabbs Foundation, Birmingham, United Kingdom and the Henry J Predolin Foundation, USA.

\section{REFERENCES}

1. Kumar SK, Rajkumar SV, Dispenzieri A, Lacy MQ, Hayman SR, Buadi FK, et al. Improved survival in multiple myeloma and the impact of novel therapies. Blood. 2008; 111:2516-2520. [PubMed: 17975015]

2. Rajkumar SV, Hayman SR, Lacy MQ, Dispenzieri A, Geyer SM, Kabat B, et al. Combination therapy with lenalidomide plus dexamethasone (Rev/Dex) for newly diagnosed myeloma. Blood. 2005; 106:4050-4053. [PubMed: 16118317]

3. Rajkumar SV, Hayman S, Gertz MA, Dispenzieri A, Lacy MQ, Greipp PR, et al. Combination therapy with thalidomide plus dexamethasone for newly diagnosed myeloma. J Clin Oncol. 2002; 20:4319-4323. [PubMed: 12409330]

4. Rajkumar SV, Jacobus S, Callander NS, Fonseca R, Vesole DH, Williams ME, et al. Lenalidomide plus high-dose dexamethasone versus lenalidomide plus low-dose dexamethasone as initial therapy for newly diagnosed multiple myeloma: an open-label randomised controlled trial. Lancet Oncol. 2010; 11:29-37. [PubMed: 19853510]

5. Rajkumar SV, Blood E, Vesole D, Fonseca R, Greipp PR. Eastern Cooperative Oncology G Phase III clinical trial of thalidomide plus dexamethasone compared with dexamethasone alone in newly diagnosed multiple myeloma: a clinical trial coordinated by the Eastern Cooperative Oncology group. J Clin Oncol. 2006; 24:431-436. [PubMed: 16365178]

6. Mikhael JR, Belch AR, Prince HM, Lucio MN, Maiolino A, Corso A, et al. High response rate to bortezomib with or without dexamethasone in patients with relapsed or refractory multiple myeloma: results of a global phase 3b expanded access program. Br J Haematol. 2009; 144:169175. [PubMed: 19036114]

7. Kumar S, Rajkumar SV. Thalidomide and lenalidomide in the treatment of multiple myeloma. Eur J Cancer. 2006; 42:1612-1622. [PubMed: 16750621]

8. San Miguel JF, Schlag R, Khuageva NK, Dimopoulos MA, Shpilberg O, Kropff M, et al. Bortezomib plus melphalan and prednisone for initial treatment of multiple myeloma. N Engl J Med. 2008; 359:906-917. [PubMed: 18753647] 
9. Cavo M, Tacchetti P, Patriarca F, Petrucci MT, Pantani L, Galli M, et al. Bortezomib with thalidomide plus dexamethasone compared with thalidomide plus dexamethasone as induction therapy before, and consolidation therapy after, double autologous stem-cell transplantation in newly diagnosed multiple myeloma: a randomised phase 3 study. Lancet. 2010; 376:2075-2085. [PubMed: 21146205]

10. Palumbo A, Falco P, Corradini P, Falcone A, Di Raimondo F, Giuliani N, et al. Melphalan, prednisone, and lenalidomide treatment for newly diagnosed myeloma: a report from the GIMEMA--Italian Multiple Myeloma Network. J Clin Oncol. 2007; 25:4459-4465. [PubMed: 17785703]

11. Palumbo A, Gay F, Bringhen S, Falcone A, Pescosta N, Callea V, et al. Bortezomib, doxorubicin and dexamethasone in advanced multiple myeloma. Ann Oncol. 2008; 19:1160-1165. [PubMed: 18326520]

12. Kumar SK, Flinn I, Noga SJ, Hari P, Rifkin R, Callander N, et al. Bortezomib, dexamethasone, cyclophosphamide and lenalidomide combination for newly diagnosed multiple myeloma: phase 1 results from the multicenter EVOLUTION study. Leukemia. 2010; 24:1350-1356. [PubMed: 20508619]

13. Weber DM, Chen C, Niesvizky R, Wang M, Belch A, Stadtmauer EA, et al. Lenalidomide plus dexamethasone for relapsed multiple myeloma in North America. N Engl J Med. 2007; 357:21332142. [PubMed: 18032763]

14. Palumbo A, Hajek R, Delforge M, Kropff M, Petrucci MT, Catalano J, et al. Continuous lenalidomide treatment for newly diagnosed multiple myeloma. N Engl J Med. 2012; 366:17591769. [PubMed: 22571200]

15. McCarthy PL, Owzar K, Hofmeister CC, Hurd DD, Hassoun H, Richardson PG, et al. Lenalidomide after stem-cell transplantation for multiple myeloma. N Engl J Med. 2012; 366:1770-1781. [PubMed: 22571201]

16. Attal M, Lauwers-Cances V, Marit G, Caillot D, Moreau P, Facon T, et al. Lenalidomide maintenance after stem-cell transplantation for multiple myeloma. N Engl J Med. 2012; 366:17821791. [PubMed: 22571202]

17. Kaplan EMP. Non-parametric estimation from incomplete observations. J Am Stat Assoc. 1958; 53:457-481.

18. Richardson PG, Weller E, Lonial S, Jakubowiak AJ, Jagannath S, Raje NS, et al. Lenalidomide, bortezomib, and dexamethasone combination therapy in patients with newly diagnosed multiple myeloma. Blood. 2010; 116:679-686. [PubMed: 20385792]

19. Khan ML, Reeder CB, Kumar SK, Lacy MQ, Reece DE, Dispenzieri A, et al. A comparison of lenalidomide/dexamethasone versus cyclophosphamide/lenalidomide/ dexamethasone versus cyclophosphamide/bortezomib/dexamethasone in newly diagnosed multiple myeloma. $\mathrm{Br} \mathbf{J}$ Haematol. 2012; 156:326-333. [PubMed: 22107129]

20. Palumbo A, Bringhen S, Caravita T, Merla E, Capparella V, Callea V, et al. Oral melphalan and prednisone chemotherapy plus thalidomide compared with melphalan and prednisone alone in elderly patients with multiple myeloma: randomised controlled trial. Lancet. 2006; 367:825-831. [PubMed: 16530576]

21. Wijermans P, Schaafsma M, Termorshuizen F, Ammerlaan R, Wittebol S, Sinnige H, et al. Phase III study of the value of thalidomide added to melphalan plus prednisone in elderly patients with newly diagnosed multiple myeloma: the HOVON 49 study. J Clin Oncol. 2010; 28:3160-3166. [PubMed: 20516439]

22. Palumbo A, Bringhen S, Rossi D, Cavalli M, Larocca A, Ria R, et al. Bortezomib-melphalanprednisone-thalidomide followed by maintenance with bortezomib-thalidomide compared with bortezomib-melphalan-prednisone for initial treatment of multiple myeloma: a randomized controlled trial. J Clin Oncol. 2010; 28:5101-5109. [PubMed: 20940200]

23. Hulin C, Facon T, Rodon P, Pegourie B, Benboubker L, Doyen C, et al. Efficacy of melphalan and prednisone plus thalidomide in patients older than 75 years with newly diagnosed multiple myeloma: IFM 01/01 trial. J Clin Oncol. 2009; 27:3664-3670. [PubMed: 19451428]

24. Rajkumar SV. Treatment of myeloma: cure vs control. Mayo Clin Proc. 2008; 83:1142-1145. [PubMed: 18828974] 
25. Kapoor P, Fonseca R, Rajkumar SV, Sinha S, Gertz MA, Stewart AK, et al. Evidence for cytogenetic and fluorescence in situ hybridization risk stratification of newly diagnosed multiple myeloma in the era of novel therapie. Mayo Clin Proc. 2010; 85:532-537. [PubMed: 20511484]

26. Avet-Loiseau H, Soulier J, Fermand JP, Yakoub-Agha I, Attal M, Hulin C, et al. Impact of highrisk cytogenetics and prior therapy on outcomes in patients with advanced relapsed or refractory multiple myeloma treated with lenalidomide plus dexamethasone. Leukemia. 2010; 24:623-628. [PubMed: 20072152]

27. Dimopoulos MA, Richardson PG, Brandenburg N, Yu Z, Weber DM, Niesvizky R, et al. A review of second primary malignancy in patients with relapsed or refractory multiple myeloma treated with lenalidomide. Blood. 2012; 119:2764-2767. [PubMed: 22323483]

28. Dong C, Hemminki K. Second primary neoplasms among 53159 haematolymphoproliferative malignancy patients in Sweden, 1958-1996: a search for common mechanisms. Br J Cancer. 2001; 85:997-1005. [PubMed: 11592772]

29. Mailankody S, Pfeiffer RM, Kristinsson SY, Korde N, Bjorkholm M, Goldin LR, et al. Risk of acute myeloid leukemia and myelodysplastic syndromes after multiple myeloma and its precursor disease (MGUS). Blood. 2011; 118:4086-4092. [PubMed: 21795746] 


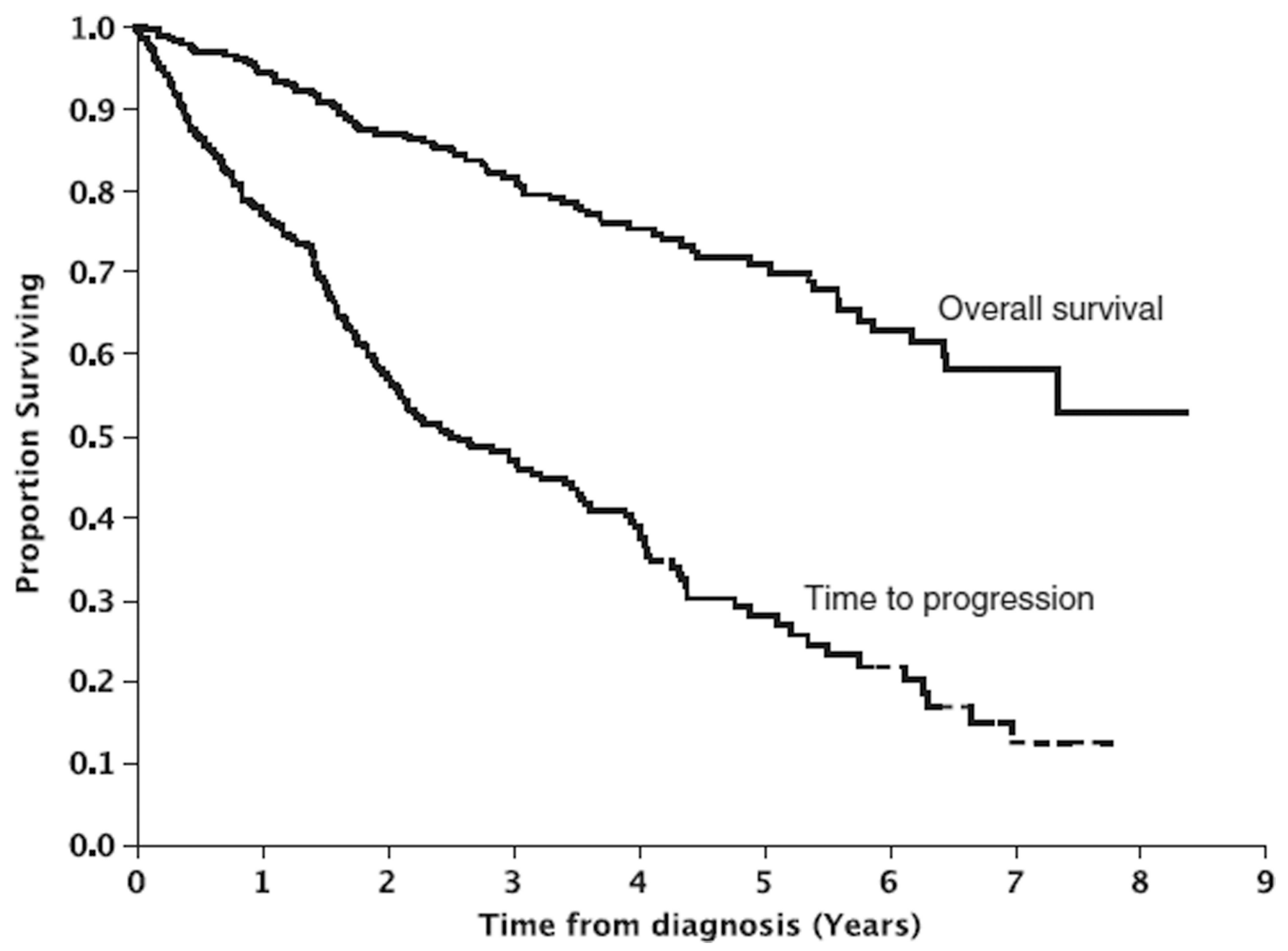

Figure 1.

Kaplan-Meier curves for OS and time to progression for all patients in study. Estimated 3 year and 5-year OS were $84 \%$ and $67 \%$, respectively. 


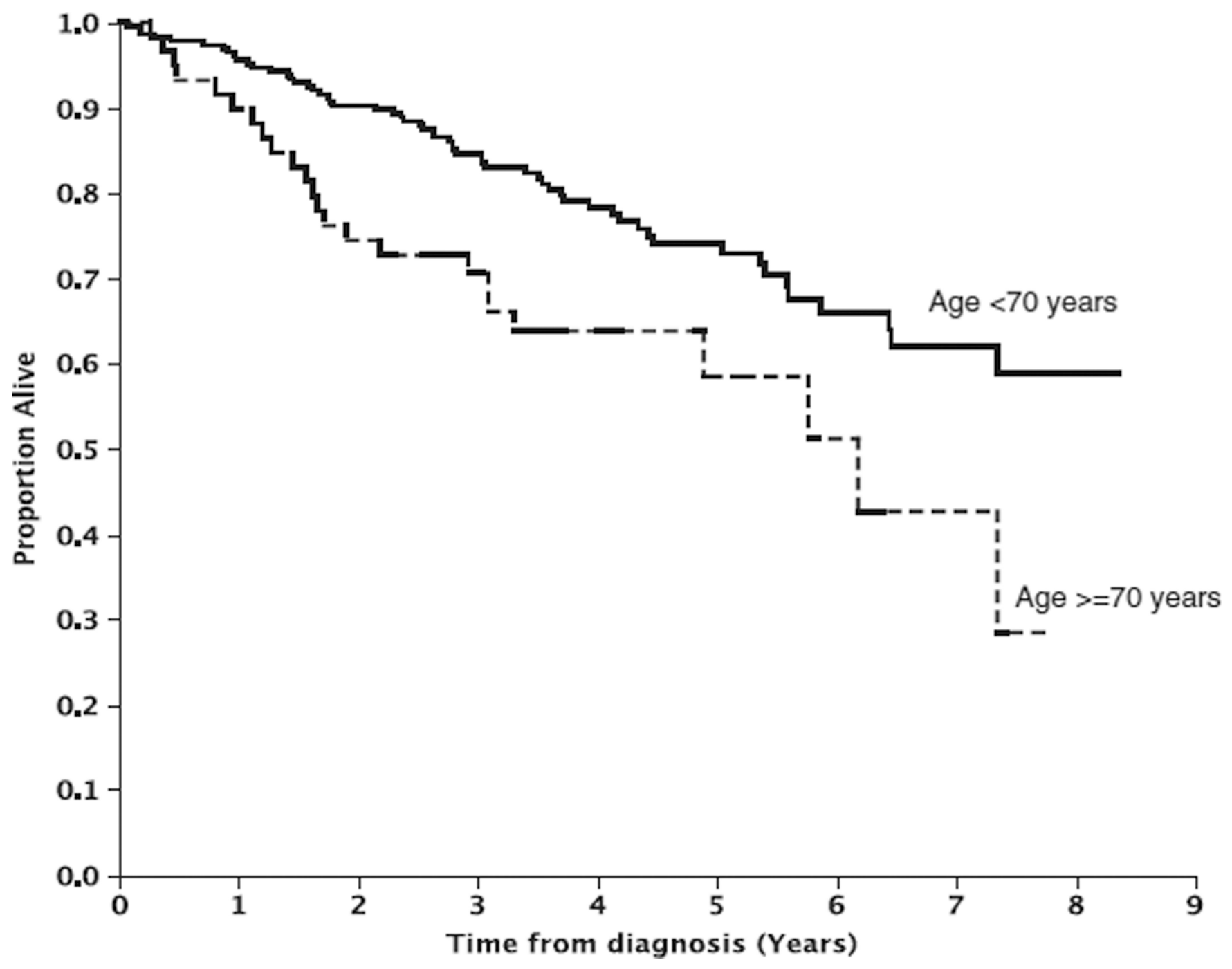

Figure 2.

Kaplan-Meier curves comparing OS among patients less than 70 years ( $n=227$ with median OS not reached) and more than 70 years ( $n=59$ with median OS of 5.8 years) with $P=0.002$. 


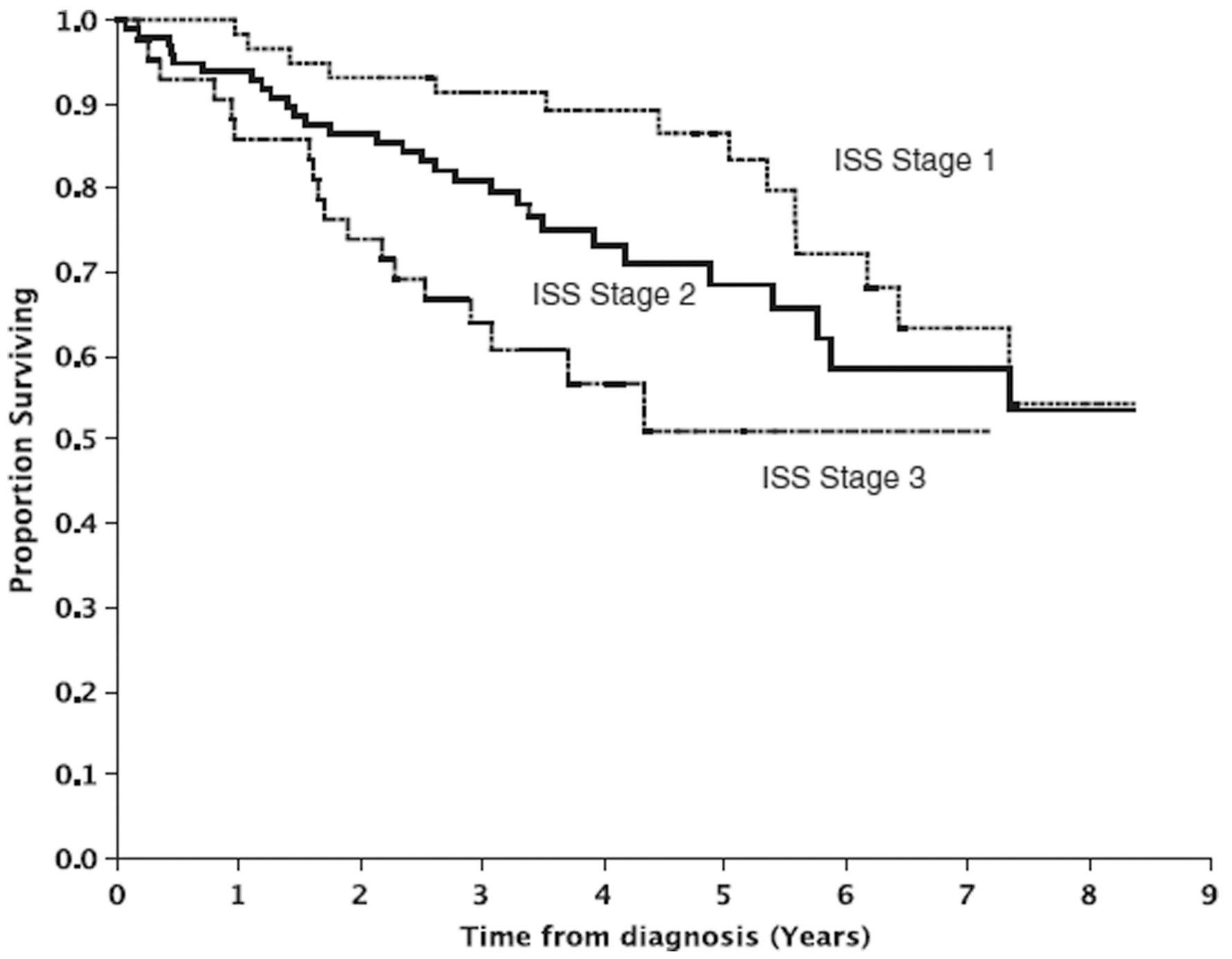

Figure 3.

Kaplan-Meier curves comparing OS among International Staging System (ISS) stage I to III. 5-year OS estimate for patients in ISS stage 1, 2 and 3 were 82, 65, and 44 months. 

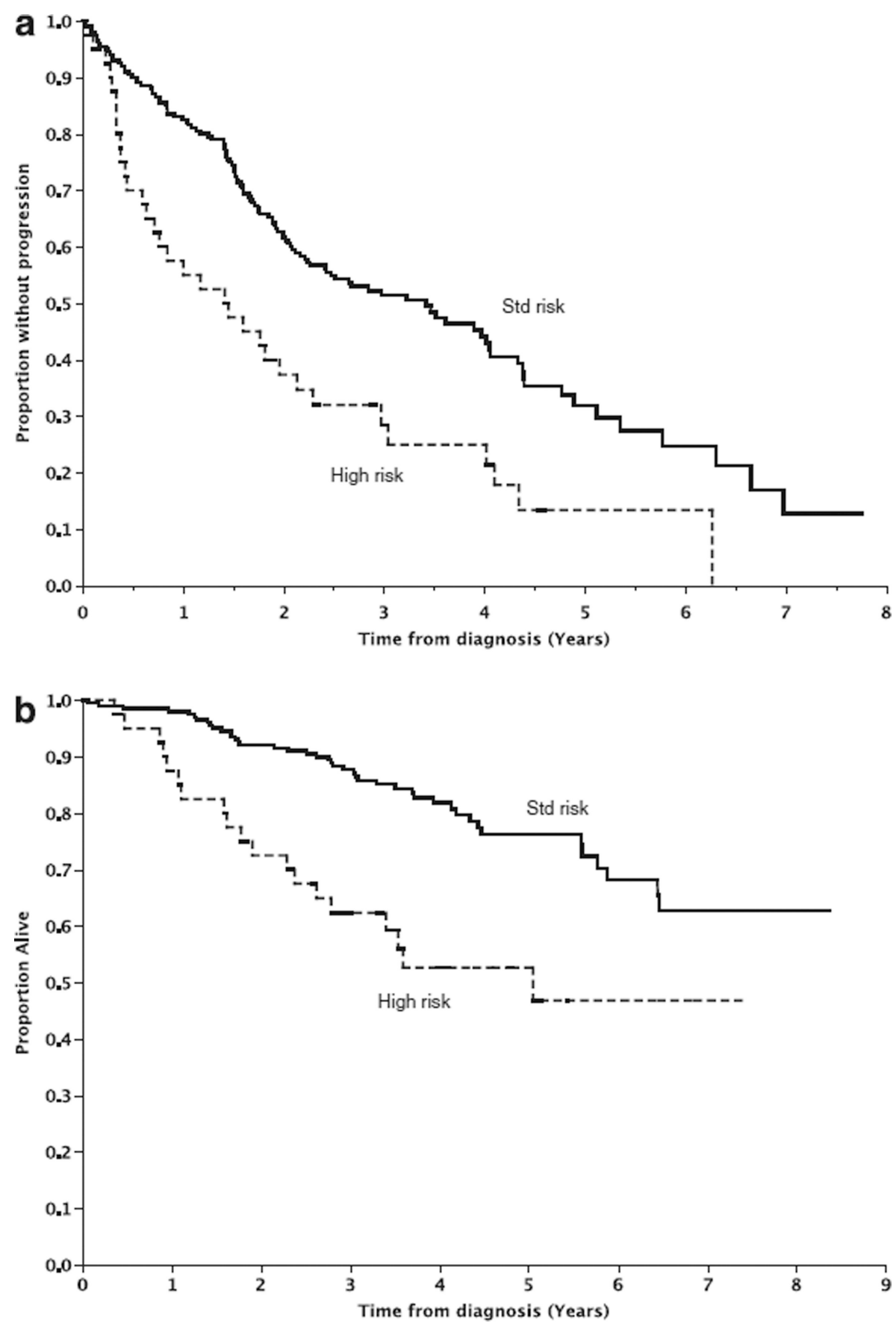

Figure 4.

(a) Kaplan-Meier curves comparing PFS among patients with high-risk cytogenetics (with median PFS of 1.4 years) as compared with standard risk (median PFS of 3.4 years) with $P=0.0002$. (b) Kaplan-Meier curves comparing OS among patients with high-risk cytogenetics (median OS of 5 years) as compared with standard risk (median OS not reached) with $P=0.0001$. 
Table 1

Baseline characteristics at diagnosis

\begin{tabular}{|lc|}
\hline Factor $(N)$ & Median (range) \\
\hline Age & $63(28-92)$ \\
Gender & \\
Male & $175(61 \%)$ \\
Female & $111(39 \%)$ \\
ISS stage ( $n=197)$ & \\
I & $58(30 \%)$ \\
II & $97(49 \%)$ \\
III & $42(21 \%)$ \\
Hgb g/dl ( $n=230)$ & $11.5(6.1-16)$ \\
Platelet $(n=210)$ & $228(69-535)$ \\
Beta 2 microglobulin $(n=205)(\mathrm{mg} / \mathrm{l})$ & $3.45(1.24-25.6)$ \\
Albumin $\mathrm{g} / \mathrm{dl}(n=217)$ & $3.6(2.1-4.8)$ \\
C-reactive protein $(n=158)$ & $0.3(0.016-47.7)$ \\
LDH IU $(n=202)$ & $158(88-509)$ \\
Calcium mg/dl ( $n=224)$ & $9.6(7.6-14.7)$ \\
Serum creatinine (mg/dl) $(n=225)$ & $1(0.5-7.9)$ \\
Plasma cell labeling index $(\%)(n=167)$ & $0.8(0-11)$ \\
$\%$ Plasma cells on bone marrow biopsy $(n=254)$ & $49(2-100)$ \\
Serum M-spike $(n=193)(\mathrm{mg} / \mathrm{dl})$ & $2.9(0.01-7.7)$ \\
\hline
\end{tabular}

Abbreviations: Hgb, hemoglobin; ISS, International Staging System; LDH IU, lactate dehydrogenase International unit. 
Table 2

Response to therapy

\begin{tabular}{|lcc|}
\hline & $\begin{array}{c}\text { Entire cohort } \\
(\mathrm{n}=286)\end{array}$ & $\begin{array}{c}\text { On lenalidomide }>12 \\
\text { months }(\mathrm{n}=93)\end{array}$ \\
\hline Objective response $(\Psi \mathrm{PR}, \%)$ & 72 & 87 \\
PR $(\%)$ & 46 & 43 \\
VGPR $(\%)$ & 14 & 20 \\
CR $(\%)$ & 11 & 23 \\
\hline
\end{tabular}

Abbreviations: CR, complete response; PR, partial response; VGPR, very good partial response. 
Table 3

Second malignancies among patient with MM (n=286)

\begin{tabular}{|lcc|}
\hline $\begin{array}{l}\text { Type of second } \\
\text { malignancy }\end{array}$ & $\begin{array}{c}\text { After initiation of } \\
\text { lenalidomide }\end{array}$ & $\begin{array}{c}\text { Before diagnosis } \\
\text { of } M M\end{array}$ \\
\hline Skin (BCC/SCC) & 9 & 12 \\
Solid tumor total ${ }^{2}$ & 10 & 37 \\
Prostate & 1 & 16 \\
Breast & 2 & 7 \\
Melanoma & 3 & 1 \\
Other solid tumors $b$ & 4 & 11 \\
Renal cell cancer & 0 & 2 \\
Hematologic total & 2 & 3 \\
AML & 2 & 0 \\
CLL & 0 & 2 \\
DLBCL & 0 & 1 \\
Total overall & 21 & 52 \\
\hline
\end{tabular}

Abbreviations: AMl, acute myeloid leukemia; BCC, basal cell carcinoma; CLL, chronic lymphoid leukemia; DLBCL, diffuse large B-cell lymphoma; SCC, squamous cell carcinoma.

${ }^{a}$ Solid tumor excludes skin cancers (except melanoma) and hematological malignancies.

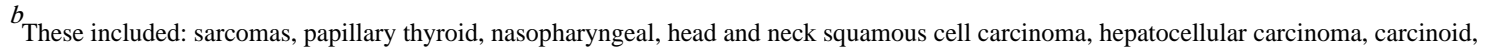
testicular cancer and bladder carcinoma. 